\title{
Infrared Air Turbine Dental Handpiece Rotor Fault Diagnosis with Convolutional Neural Network
}

\author{
Yi-Cheng Huang* and Pin-Jun Wang \\ Department of Mechatronics Engineering, National Changhua University of Education, \\ No. 1, Chinde Rd., Changhua City, Taiwan
}

(Received December 23, 2019; accepted April 30, 2020)

Keywords: deep learning, convolutional neural networks, dental handpieces, infrared thermal imaging

AI has been widely used this century. In this study, we demonstrated deep learning in a convolutional neural network (CNN). CNNs are often used for image recognition and image classification. A noninvasive infrared thermal imaging camera was used for the diagnosis of damage in dental handpiece rotors. Areas in a thermal image were considered as specific conditions, which can simplify the detection of complex physical conditions. A CNN was trained to detect thermal images. Six sets of experiments were performed on rotor thermal imaging for $30 \mathrm{~s}$ and $1 \mathrm{~min}$ at 15, 20, and $25 \mathrm{psi}$ air pressures. The thermal image shooting speed was 5 frame/s. Each thermal image map was subjected to CNN training. An accuracy curve was observed to evaluate the performance of the model, where the closer the accuracy variable is to 1 , the more accurate the model is. The experimental results proved that the accuracy of idling at 25 psi was $100 \%$. The proposed system can diagnose the rotor condition automatically.

\section{Introduction}

Dental handpieces are medical devices that are commonly used by dentists. Problems with an internal rotor bearing are responsible for $70 \%$ of the faults in typical dental handpieces. Dental handpieces require regular maintenance and immediate replacement when damaged. Dentists must avoid using devices with damaged bearings to treat patients. Therefore, the quality of dental handpieces is crucial. The function of a dental handpiece involves pumping high-pressure gas into the handpiece chamber, which forms an air flow that rotates microturbine blades and drives the rotor. The speed can be changed by changing the pressure of the air and the shape of the rotor blades. Although the handpieces can be diagnosed with sound signals, this is not easy because of the noisy background of the environment.

A typical dentist may be oblivious to any internal damage that might occur to a dental handpiece during a clinical treatment. High-speed air turbines in dental handpieces rotate at $>300000 \mathrm{rpm}$. A difference in air pressure or bearing damage can affect their speed. A damaged rotor experiences more friction and its speed decreases owing to the imbalance

*Corresponding author: e-mail: ychuang@cc.ncue.edu.tw

https://doi.org/10.18494/SAM.2020.2755 
produced at high temperatures during cutting. These high temperatures can be conducted to dental tools. Water injection cannot reduce such temperatures, and teeth can be damaged as a result.

According to the law of blackbody radiation, objects with temperatures above absolute zero have infrared radiation. All thermal radiation is composed of infrared with different frequencies. Figures 1 and 2 are respectively thermal images of normal and damaged rotors for drilling teeth without water injection and cooling. In this work, an infrared thermal imager was used to study a dental handpiece rotor. A convolutional neural network (CNN) was applied to each infrared thermal image to determine the rotor damage. For rotor fault diagnosis, thermal images of the rotor under idling and drilling conditions were captured. Zach and Cohen ${ }^{(1)}$ studied the severity of dental pulp lesions caused by heat. If the amount of water injected during the treatment is insufficient, the heat generated by friction during cutting cannot be reduced. During the drilling of teeth, heat is transferred to the dentin, which in turn affects the pulp and produces lesions. Widodo et al. ${ }^{(2)}$ obtained vibration signals and thermal image maps of different types of fault from a machine fault simulator by using self-organizing map (SOM) training for intelligent machine diagnostics. The experimental results indicated that the thermal images provided a reasonable result for intelligent machine fault diagnosis. Liu et al. ${ }^{(3)}$ operated on five types of servers. A thermal image of heat distribution was captured on the surface of the server by using a thermographic camera. A thermal image matrix was then input into the support vector machine (SVM) for training. The accuracy of SVM training results was $91.11 \%$, which confirmed that the surface temperature could be photographed by using the thermographic camera to investigate internal damage. Janssens et al. ${ }^{(4)}$ used thermal images for deep learning to automatically recognize machine status. The heat source was different for different faults and locations. In their thermal image analysis of fault detection and in oil level prediction of rotating machinery, the accuracy rates were 95 and $91.67 \%$, respectively. It was found that crucial areas in the thermal image can be regarded as specific conditions. Therefore, the training of CNNs with thermal images can facilitate the detection of complex sensor data.

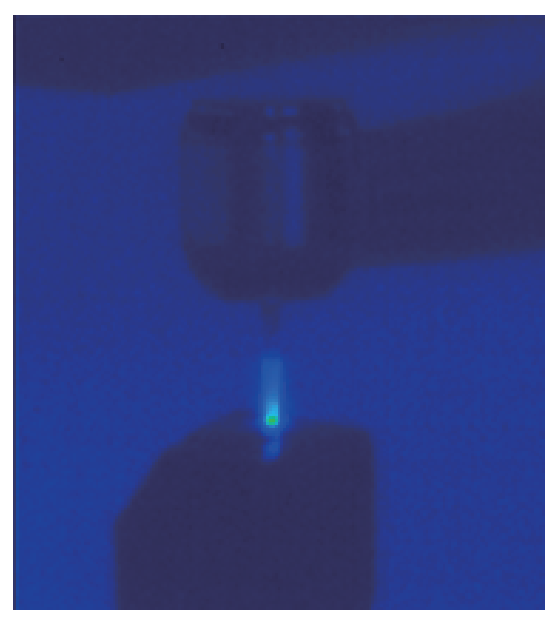

Fig. 1. (Color online) Thermal image of normal rotor dental drill.

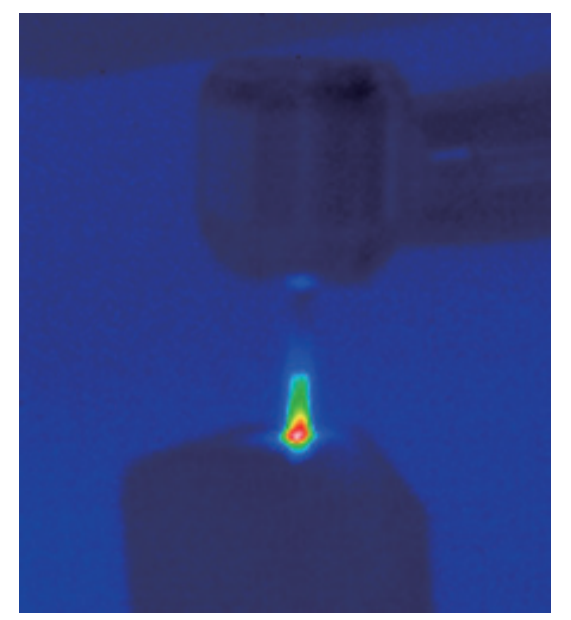

Fig. 2. (Color online) Thermal image of damaged rotor dental drill. 
Thus, a CNN can detect different fault conditions in a rotating machine without physical knowledge.

\section{CNN}

LeCun et al. invented the type of deep artificial neural networks called CNNs. ${ }^{(5)}$ A CNN can extract specific features or patterns as a training set from numerous images. CNNs can process the vectors of $2 \mathrm{D}$ arrays, such as language, image, audio, and vibration signals. In particular, many image recognition architectures and models are based on CNNs. A typical $\mathrm{CNN}$ is mainly composed of an input layer, a convolution layer, a pooling layer, a fully connected layer, and an output layer. Each layer plays a different role. Figure 3 shows a typical CNN.

\subsection{Convolution layer}

The convolution layer requires some parameters, such as kernel size, stride, padding, dimensions, and the number of feature maps. The kernel size is the size of a filter. The stride is the step size of the filter when it moves. The padding makes the size of the graphical output equal to that of the input. Neurons can be set to zero, except for in the original picture. In the present study, when the filter size was $(f w, f h)$, the original picture size was $(w, h)$, and the output size was $(w-f w+1, h-f h+1)$, and when padding was performed around the original image, $N$ was the number of paddings and the output size was $(w+2 N-f w+1, h+2 N-f h+1)$. The purpose of the convolution operation was to extract different features and local regions of an image. The image was generated by the calculation of the filter to produce a new feature map, which can emphasize different features. Different filters were used to generate different feature maps corresponding to different feature extractions. Multilayered calculations can extract more complex features. The size of the output was calculated using Eq. (1).

$$
\text { Outputshape }=\frac{\text { Inputshape }- \text { Filtershape }+2 \times \text { padding }}{\text { stride }}+1
$$

stride: Filter step size

padding: Number of paddings

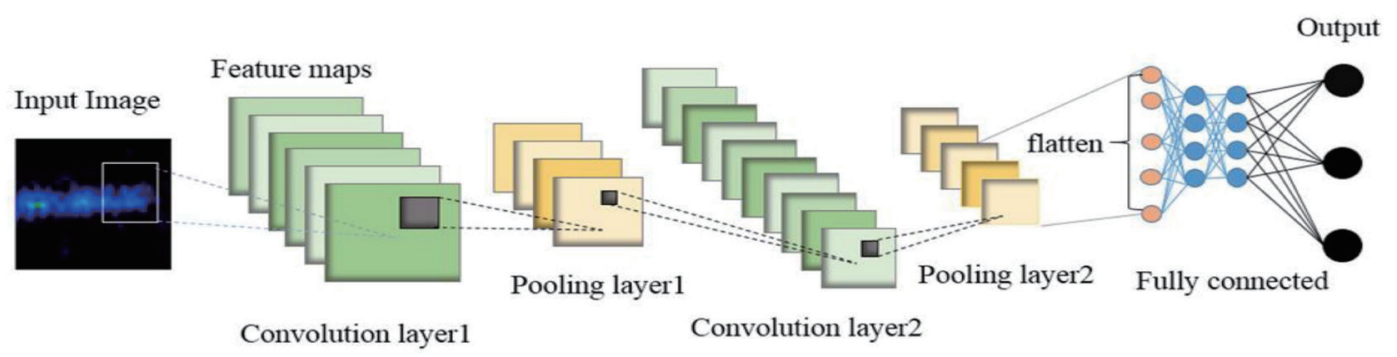

Fig. 3. (Color online) Layers of a CNN. 


\subsection{Pooling layer}

The pooling layer was the subsampling result of the feature map. For example, when calculating the size of $4 \times 4$ pixels through pooling, the output obtained is $2 \times 2$ pixels. The most common method is to choose the minimum or maximum values from one of the $4 \times 4$ pixels. The pooling layer can retain the largest feature of the image to reduce its size. The pooling layer reduces the size of the training set and the training time. It can also prevent the overfitting of the network. Figure 4 illustrates the pooling method.

\subsection{Fully connected layer}

Both the convolution layer and the pooling layer extracted features. The fully connected layer of the input neural network was used for classification. Each neuron was connected to upper neurons, as illustrated in Fig. 5. There was no sharing of any link, and the output prediction was judged using the feature map of the same layer.

\section{Activation Function}

Equation (2) defines the activity function. When the activation function was positive, the neuron was activated to allow it to pass to the next adjacent neuron. Nonlinear functions were selected as activation functions for hidden layer nodes, such as ReLU, tanh, and sigmoid functions. Because the output value of the neuron was an arbitrary value, the output value could be simplified to a number between 0 and 1 after applying the function, increasing the efficiency of model training. $x_{i}$ is the input value for each neuron and $w_{i}$ is the weight of the connection of the outputs of the ith neuron to itself.

$$
f(x)=\sum_{i} w_{i} x_{i}+b
$$

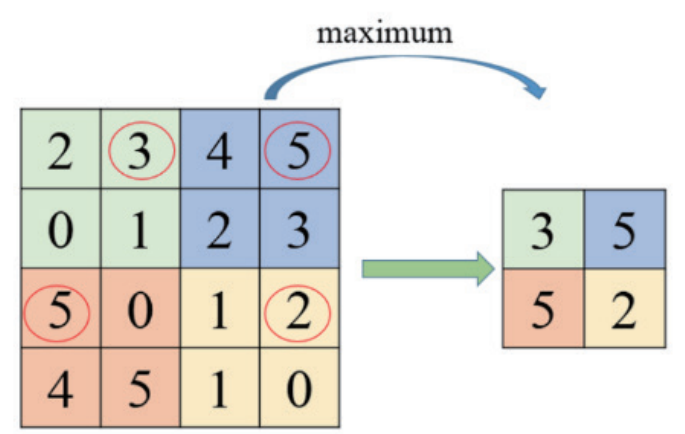

Fig. 4. (Color online) Maximum pooling operation.

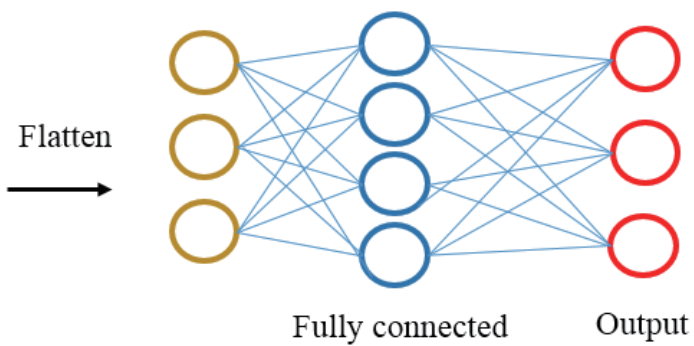

Fig. 5. (Color online) Schematic of the fully connected layer. 


\subsection{ReLU function}

ReLU is the most commonly used activation function for artificial neural networks and is given by Eq. (3). It can represent a valid function from zero to infinity. Krizhevsky et al. ${ }^{(6)}$ reported that the training of deep convolutional networks of ReLU functions was faster than that of tanh functions. A comparison of convergence speeds is presented in Fig. 6.

$$
f(x)=\max (0, x)
$$

\subsection{Softmax function}

The softmax function was used in a multiclassification model to calculate the probability distribution of different events. The probability can help determine the target category of the input. The softmax function outputs a probability in the range of 0 to 1 , and the sum of the probabilities is 1, as presented in Eq. (4), wherein $y_{q k}$ represents the team's $q$ th input $x_{q}$ belonging to the estimation probability of class $k$ and $p$ is the number of output nodes.

$$
y_{q k}=\frac{e^{S_{q k}}}{\sum_{b=1}^{p} e^{S_{q b}}}
$$

\subsection{Categorical cross entropy (CCE)}

In multivariate classification, the most commonly used loss function is the CCE. The output node activation function in this study is the softmax function. The cross entropy is used to estimate the probability and actual distribution of training, as presented in Eq. (5), wherein $y_{q k}$ is the output value of softmax and $d_{q k}$ is the category value of the target. When the predicted value is close to the actual value, the loss function is close to zero.

$$
C C E=-\sum_{q} \sum_{k} d_{q k} \log \left(y_{q k}\right)
$$

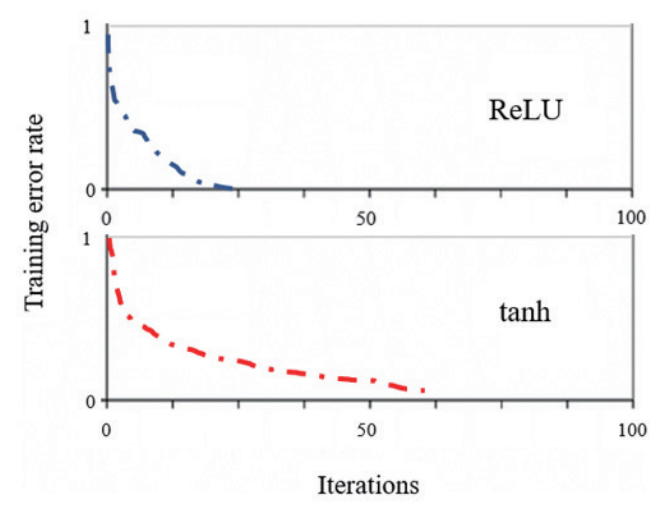

Fig. 6. Illustration for the comparison between ReLU and tanh convergence. ${ }^{(6)}$ 


\section{Experimental Setup}

We established an evaluation model by using CNNs. In this experiment, a dental handpiece was used to perform two experiments. Before using the dental handpiece, its health performance was evaluated. The dental handpiece was equipped with a normal rotor and a damaged rotor with a defective bearing, as illustrated in Fig. 7. A charge-coupled device was used to image the outer ring bearing surface. Scratches caused by the friction between the cap and bottom of the dental handpiece were observed, as shown in Fig. 8, wherein the white part is the reflection of illumination. The cage of the handpiece was subjected to centrifugal force and vibration when the dental handpiece rotated at a high speed. The extent of the damage directly affected the speed of the dental handpiece.

\subsection{Thermal image of idling}

A valve pressure controller was used to set air pressure. In idling experiments with an infrared thermal imaging camera, the air pressure was set at 15, 20, and 25 psi, as illustrated in Fig. 9. To avoid changes in the temperature due to background objects, the experimental setup

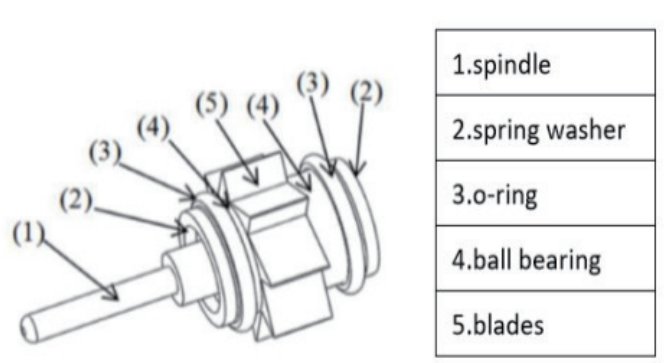

Fig. 7. Dental handpiece rotor and dental drill spindle module.

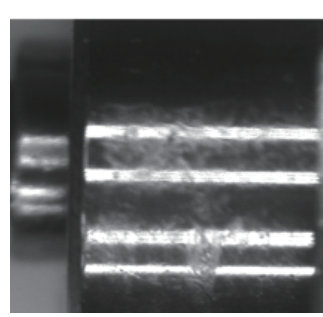

(a)

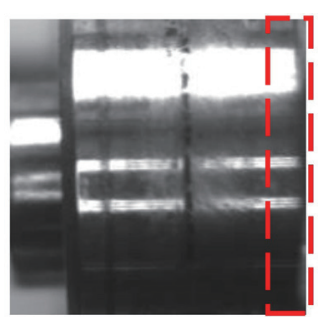

(b)
Fig. 8. (Color online) Bearing shell surface (position on number 4 surface in Fig. 7). (a) Healthy rotor. (b) Damaged rotor.

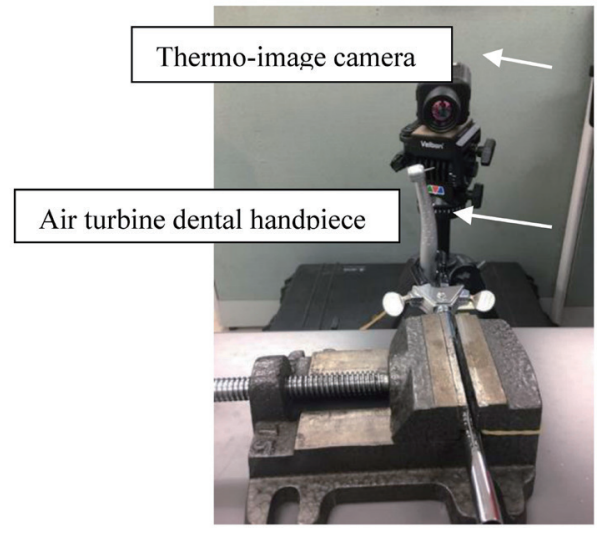

Fig. 9. (Color online) Setup of thermal imaging in idling experiment. 
was covered with a box. During the experiment, the drill of the dental handpiece was observed, which is numbered as 1 in Fig. 7, because teeth are exposed to the drill. The unbalanced rotation of the rotor produced a large amount of friction. The transfer of heat affected the temperature of the drill spindle. Two methods were used to diagnose the rotor bearing of the dental handpiece in the experiment. The first was an offline method to convert thermal image pixels to grayscale values. After each pixel takes a value of $0-255$, it can be seen that the brighter the pixel, the higher the temperature. The gray level of each pixel on the $x$ - and $y$-axes can be summed and the peak of the highest temperature position is depicted thereof. The second method is to train the CNN with thermal images by importing all the images into the $\mathrm{CNN}$ used for image recognition technology. Automatic diagnosis of the rotor bearing health of a dental handpiece should be achieved after the CNN model is deployed.

\subsection{Thermal imaging of cutting process}

Actual cutting temperatures were compared by installing healthy and damaged rotors in the dental handpiece. The dental handpiece and a bulk leucite glass-ceramic material were deployed on a desktop computer numerical control (CNC) platform, and the drilling depth was set to $0.1 \mathrm{~mm}$. The thermal images were captured without water injection, and the temperature of the cutting was observed. Figure 10 presents the experimental setup.

\subsection{Sound signals}

In this experiment, a microphone (PCB, Piezotronics) with a spectrum analyzer was used to measure the sound signals. The experimental setup is presented in Fig. 11; both the microphone and the dental handpiece are visible. We measured a new rotor, a cage-damaged rotor, and a collet-damaged rotor, all of which were used with the same dental handpiece. The idling pressure was set at 15,20, and 25 psi when measuring the idling sound signal. Subsequently, we performed a fast Fourier transform (FFT) on the signal. The rotational speed of the rotor for different degrees of health of the dental handpiece was observed.

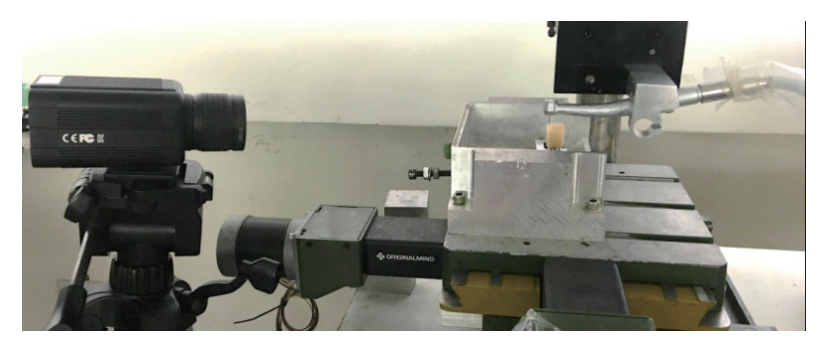

Fig. 10. (Color online) Experimental setup for thermal imaging during cutting.

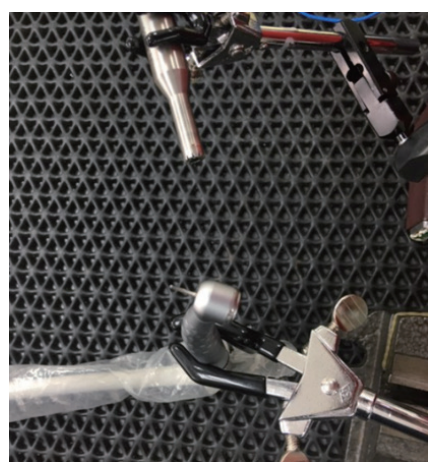

Fig. 11. (Color online) Experimental setup for sound measurement. 


\subsection{CNN structure}

The well-known Python library Keras was used to develop the CNN structure. The thermal imaging speed was 5 frame/s. A total of 310 thermal images were continuously captured to obtain the steady-state speed of the rotor and the temperature response after an initial transient state. As the hardware, a PC-based computer with an Intel Core i7-8700 processor was used with a 1080 graphics card for $\mathrm{CNN}$ training. The calculation time for training 420 pictures to determine each type of defect was about $15 \mathrm{~s}$. We compared the effect of idling times of 30 and $60 \mathrm{~s}$ on the temperature. In the $30 \mathrm{~s}$ experiment, 20 thermal images from 28 to $32 \mathrm{~s}$ were selected, and in the $60 \mathrm{~s}$ experiment, 20 thermal images from 58 to $62 \mathrm{~s}$ were selected.

Because of the small datasets, the amount of data as shown in Fig. 12 was enhanced tenfold by using a Keras ImageDataGenerator. There are many image amplification methods. We can use image translation, flipping, and zooming to perform the image amplification processes. The vertical and horizontal translation rates relative to the total height were set as $20 \%$, while the flip angle was within 20 degrees with a random $20 \%$ zoom. Panning and flipping refer to the original shooting position, and the angle while zooming refers to the distance of the shooting. Therefore, these three processing methods were used to amplify the original images to increase the size of the data training set.

After amplification, 200 images of each category were obtained and imported in the $\mathrm{CNN}$ for training. From a total of 600 thermal images, $70 \%$ (420) were selected as a training set and the remaining 30\% (180) of images were selected as a test set. The CNN had three convolutional layers, three pooling layers, three flat layers, and two fully connected layers. We used the ReLU and softmax functions as activation functions. In the experiment, we performed CNN training with six sets of data. The different conditions in the experiment are listed in Table 1.

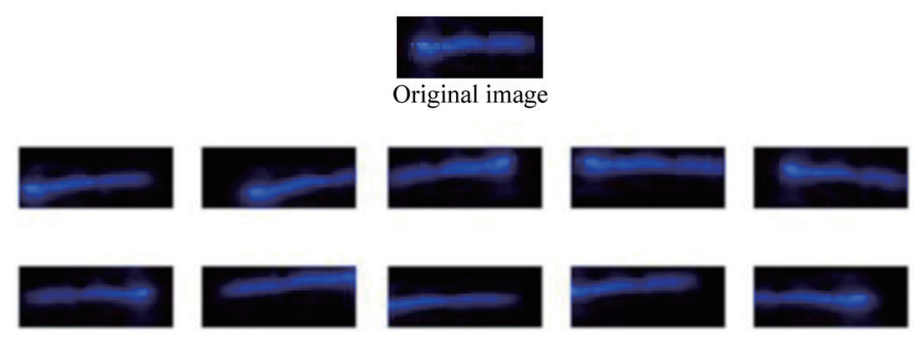

Fig. 12. (Color online) Images generated by ImageDataGenerator to increase number of images tenfold.

Table 1

Six sets of different air pressures and idling times.

\begin{tabular}{lcc}
\hline & Air pressure (psi) & Idling time (s) \\
\hline First group & 15 & 30 \\
Second group & 15 & 60 \\
Third group & 20 & 30 \\
Fourth group & 20 & 60 \\
Fifth group & 25 & 30 \\
Sixth group & 20 & 60 \\
\hline
\end{tabular}




\section{Experimental Results}

The signal was converted using an FFT, as shown in Fig. 13, to obtain the speeds of different rotors. The higher the air pressure, the higher the speed. On increasing the air pressure by $5 \mathrm{psi}$, the rotor speed increased by approximately $24000 \mathrm{rpm}$. At the same air pressure, the frequency of the damaged rotor differed from that of the new rotor by $>250 \mathrm{~Hz}$. The broken collet denoted as chuck broken, an assembly part of the dental drill and dental rotor in Fig. 7, exhibited the lowest speed. Because the sound signal is easily affected by the environment, infrared thermal imaging is used for diagnosis.

\subsection{Grayscale}

Each thermal image was converted to grayscale values of 0-255 through RGB operations. The original thermal image was $50 \times 20$ pixels and each pixel had a corresponding grayscale. A histogram of the pixels was constructed along the $x$ - and $y$-axes, as shown in Fig. 14. The position with the highest temperature can be observed from the peak.

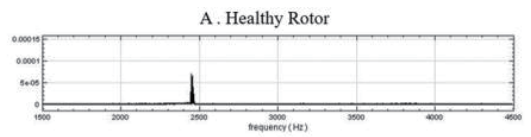

B . Cage broken Rotor

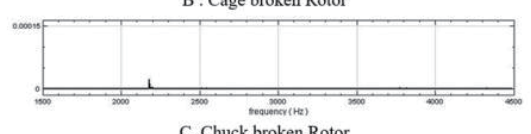

C. Chuck broken Rotor

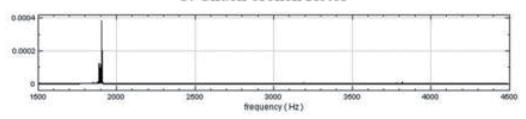

(a)
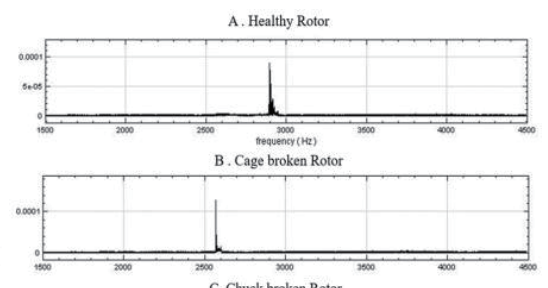

C. Chuck broken Rotor

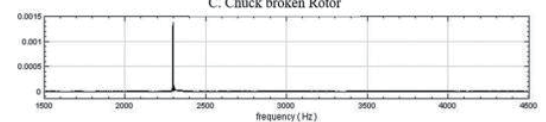

(b)
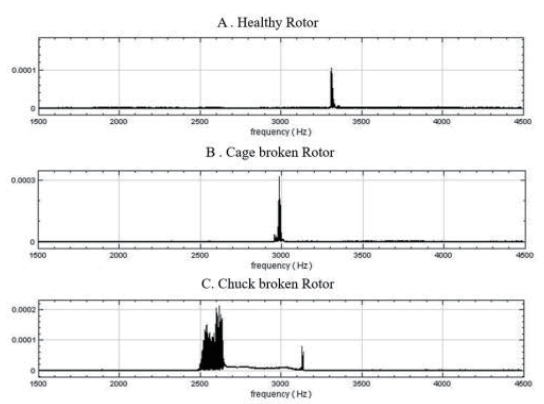

(c)

Fig. 13. FFTs of sound signal at different pressures of air for healthy, cage-broken, and collet-broken rotors. (a) 15 , (b) 20 , and (c) 25 psi.
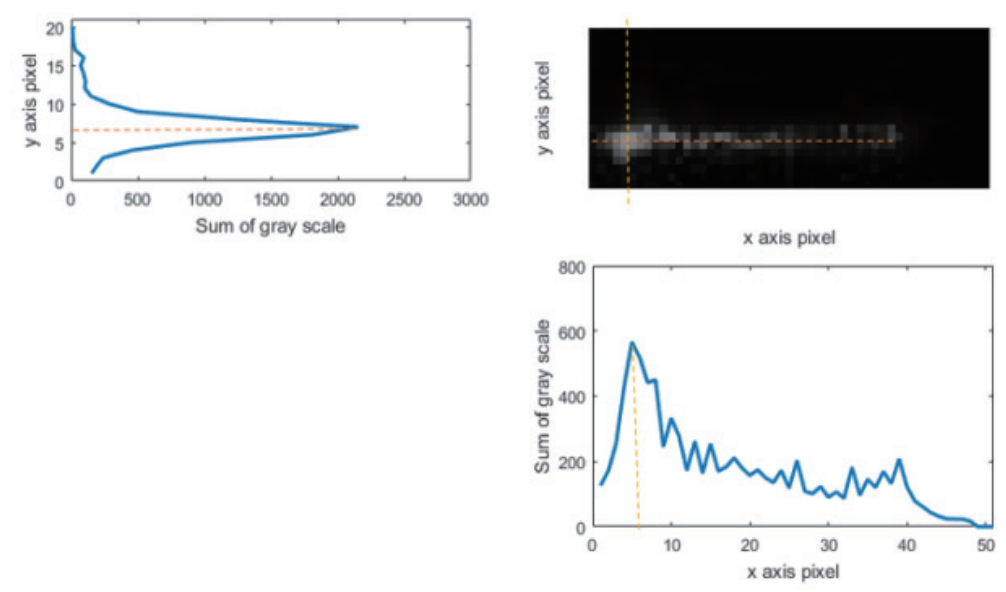

Fig. 14. (Color online) Histogram for the thermal image. 
The effects of pressures of 15, 20, and 25 psi applied for $30 \mathrm{~s}$ were compared. In Fig. 13, A denotes the healthy rotor, $\mathrm{B}$ denotes the cage-damaged rotor, and $\mathrm{C}$ denotes the collet-damaged rotor. The numbers 1, 2, and 3 represent pressures of 15, 20, and 25 psi, respectively. With increasing pressure, the temperature difference between the damaged and healthy rotors became more obvious. The result of idling at different pressures is presented in Fig. 15. The increase in the temperature was most obvious at 25 psi. Thermal images obtained with a pressure of $25 \mathrm{psi}$ can thus be used for diagnosis. However, for the thermal image for air pressure at $15 \mathrm{psi}$, the sum of the grayscale for the $x$-axis histogram of A1 (healthy rotor) is larger than those for A2 (cage-damaged rotor) and A3 (collet-damaged rotor). In contrast, at $20 \mathrm{psi}$, the sum of the grayscale for the histogram of B1 (healthy rotor) is less than that for A3 (collet-damaged rotor). Therefore, it was difficult to choose an image-processing threshold for this analysis, especially because of the complex relationship between the frictional force of the rotor with the cage and the unbalanced rotational fluidic friction heat when the dental handpiece was pressurized and operated at a very high speed. Nevertheless, the histogram can be used to find the location with the highest temperature, and the health status of the dental handpiece can be diagnosed. However, positioning errors caused by rotation, translation, and vibration of images may result in an incorrect diagnosis of the health status. Therefore, when using the CNN for training, adding rotation and translation images to the dataset of the CNN should increase the speed and accuracy of the diagnosis.

\subsection{CNN training results}

The experimental parameter of setting stride was set to two. The kernel size was $5 \times 5$. The ReLU and the softmax functions were used as activation functions. Six sets of experimental data were trained using CNNs. The training convergence can be observed using CCE. The horizontal axis was the training epoch number and the vertical axis was the cross entropy loss function, as shown in Fig. 16. When the predicted value was close to the actual value, the loss function was small. The stable trend indicated that the model converged.

The results for the six groups defined in Table 1 are illustrated in Fig. 17. In each category, 200 thermal images were trained, giving a total of 600 thermal images. In the accuracy charts in Fig. 17, the horizontal axis represents the epoch number and the vertical axis represents the accuracy rate. The closer the accuracy rate was to 1 , the higher the accuracy was.

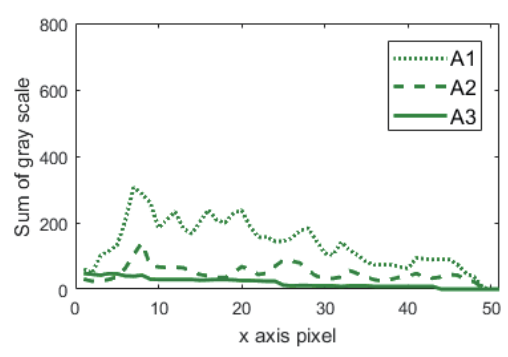

(a)

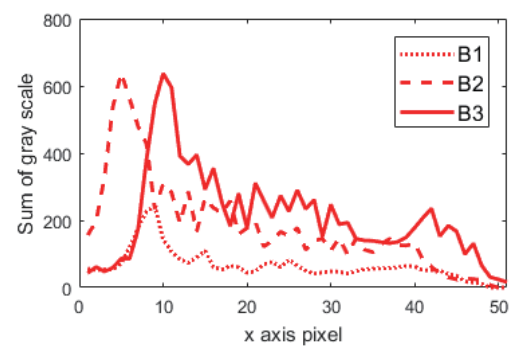

(b)

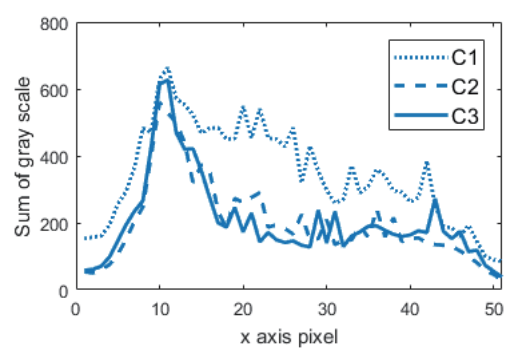

(c)

Fig. 15. (Color online) Results of idling for $30 \mathrm{~s}$ at different pressures. 


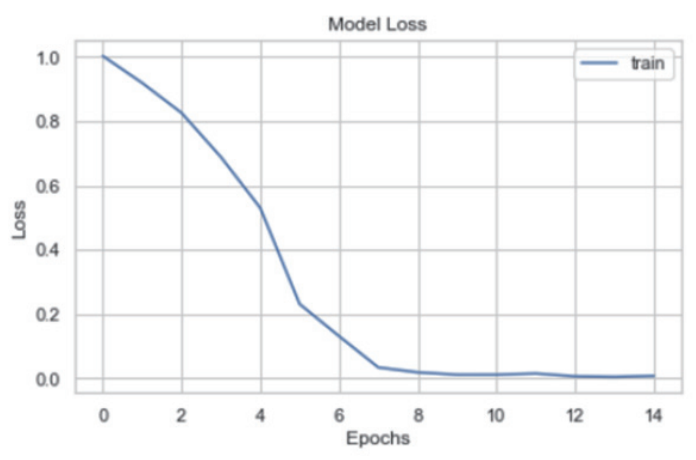

Fig. 16. (Color online) Categorical cross entropy.

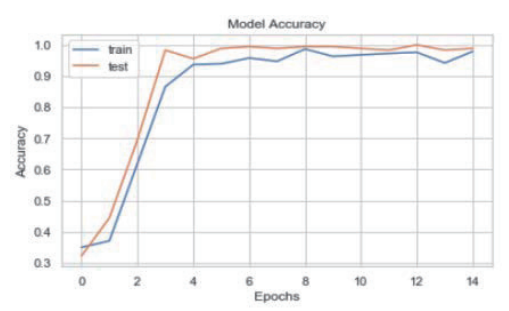

(a)

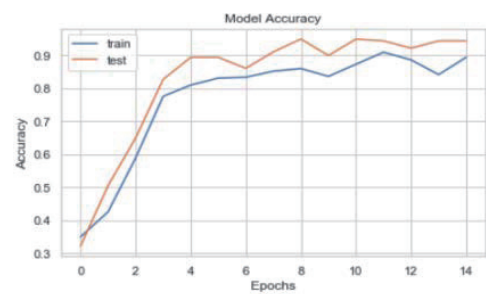

(d)

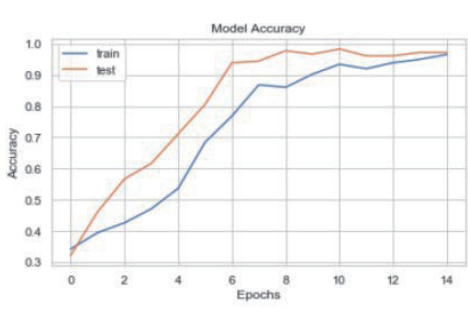

(b)

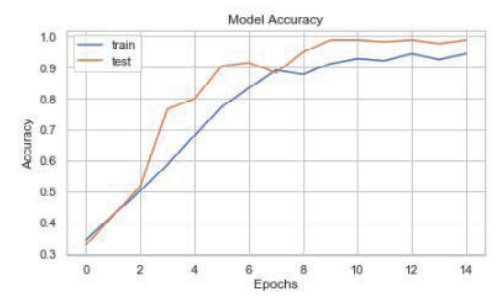

(e)

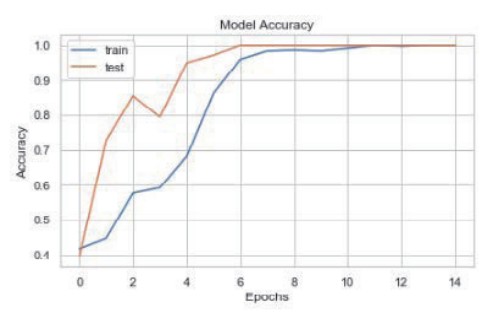

(c)

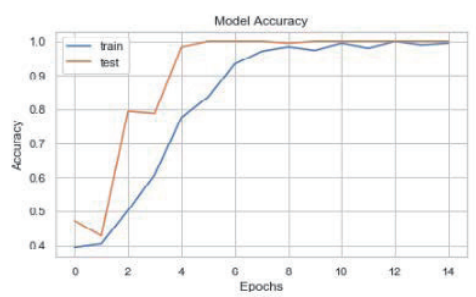

(f)

Fig. 17. (Color online) $\mathrm{CNN}$ accuracy results for six different experiments. (a) Air pressure, 15 psi; idling time, $30 \mathrm{~s}$; accuracy, 98.89\%. (b) Air pressure, 20 psi; idling time, 30 s; accuracy, 97.22\%. (c) Air pressure, 25 psi; idling time, 30 s; accuracy, 100\%. (d) Air pressure, 15 psi; idling time, 60 s; accuracy, 94.44\%. (e) Air pressure, 20 psi; idling time, 60 s; accuracy, $98.89 \%$. (f) Air pressure, 25 psi; idling time, 60 s; accuracy, $100 \%$.

The six groups of experiments for dental handpieces at different pressures were performed using CNNs. The accuracy of the thermal image plot model at 25 psi was $100 \%$. The accuracy of training at $25 \mathrm{psi}$ was $100 \%$ with convergence almost reached at the sixth iteration. The training of CNNs with thermal images can thus help to diagnose the health of the internal rotor of dental handpieces.

To observe the effects of the data size on the accuracy, the number of images in the fifth set of experimental data was manually increased 30-fold. A total of 1800 thermal images were trained using the CNN. From a total of 1800 thermal images, 70\% (1260 images) were used as the training set, whereas 30\% (540 images) were used as the test set. The effects of various data set sizes on the CNN resolution accuracy were compared. The comparison results are presented in Fig. 18. The accuracy of the fifth iteration using the amplified data was $>90 \%$. The amplification of data can be used to improve the accuracy when the accuracy is low. 
We reduced the numbers of convolution layers and pooling layers in these CNNs and observed the effect on accuracy, as shown in Fig. 19. From the results, reducing the number of layers in the CNN adversely affected the accuracy, which dropped from 100 to $98.33 \%$.

\subsection{Verification by cutting experiment}

Both healthy and cage-damaged rotors were subjected to cutting experiments, and a tooth (bulk leucite glass-ceramic material) was drilled at a cutting pressure of $30 \mathrm{psi}$ using the dental handpiece. The processing results of the thermal images are illustrated in Figs. 20 and 21. The feed rate was $6 \mathrm{~mm} / \mathrm{min}$ and the depth of the cut was $0.1 \mathrm{~mm}$. The temperature of the healthy rotor was approximately $38{ }^{\circ} \mathrm{C}$ and that of the damaged rotor was approximately $50.1{ }^{\circ} \mathrm{C}$; there is a difference of $12.1^{\circ} \mathrm{C}$. In addition, the larger the range of heat conduction, the higher the temperature and the greater the probability of tooth damage.

The histograms for the above thermal images of cutting are presented in Fig. 22. The peak for the cage-damaged rotor was considerably higher than that for the healthy rotor by 3000 grayscale, indicating the much higher temperature for the cage-damaged rotor.

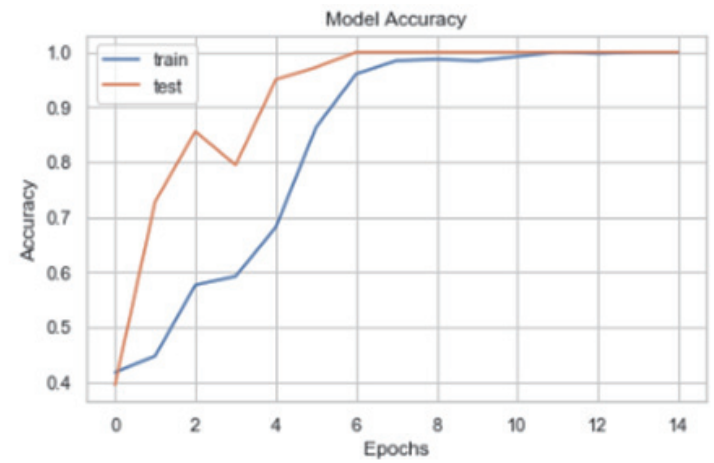

(a)

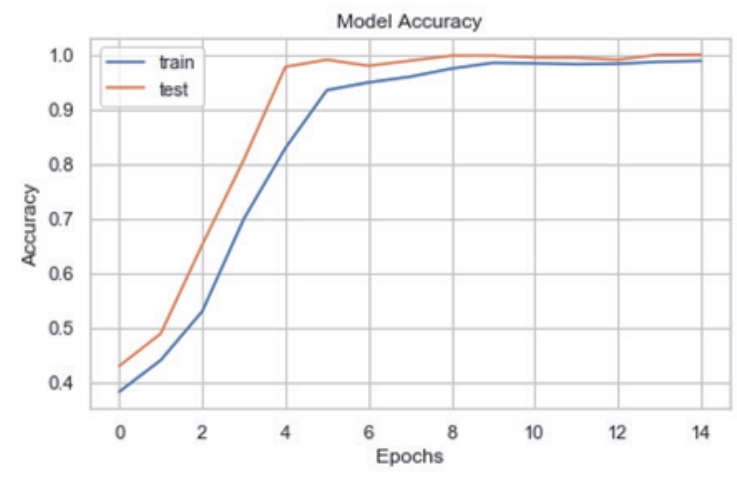

(b)

Fig. 18. (Color online) Accuracy comparison. (a) Original data accuracy. (b) Amplification data accuracy.

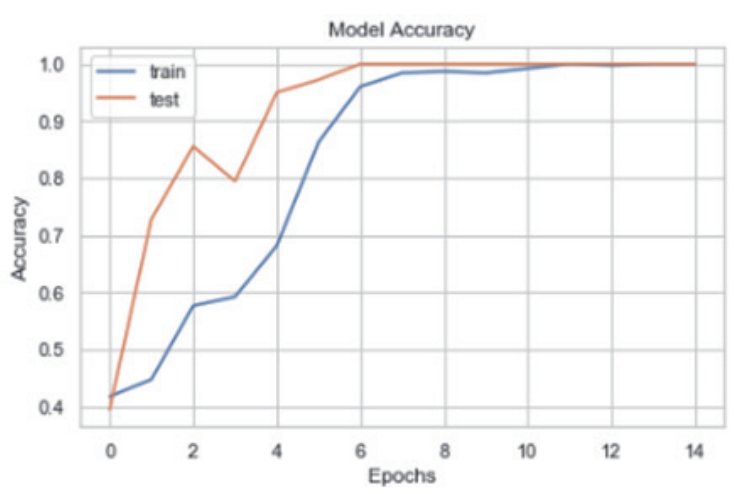

(a)

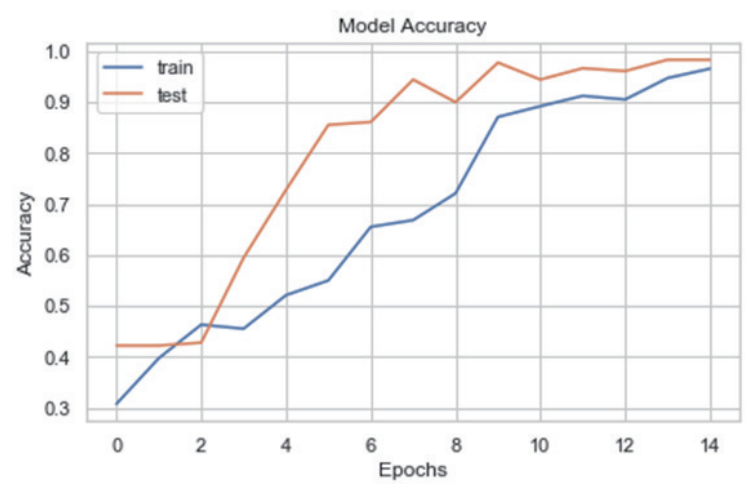

(b)

Fig. 19. (Color online) Accuracy comparison. (a) Original layer (Accuracy: 100\%). (b) Reduction layer (Accuracy: 98.33\%). 


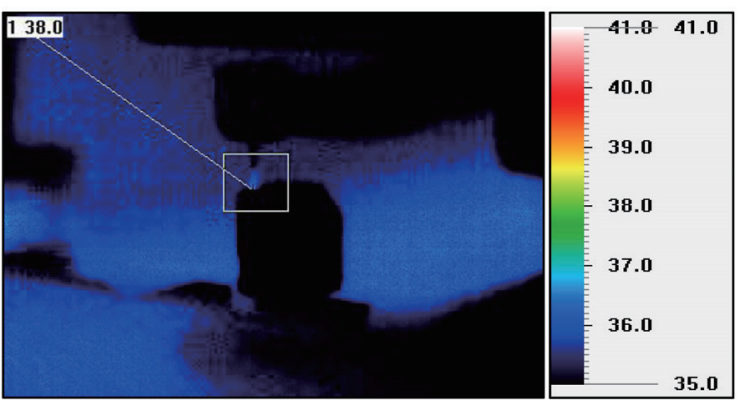

Fig. 20. (Color online) Cutting with healthy rotor.

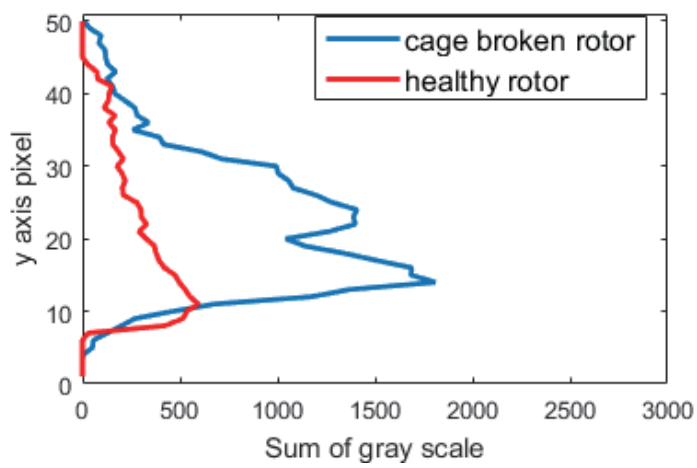

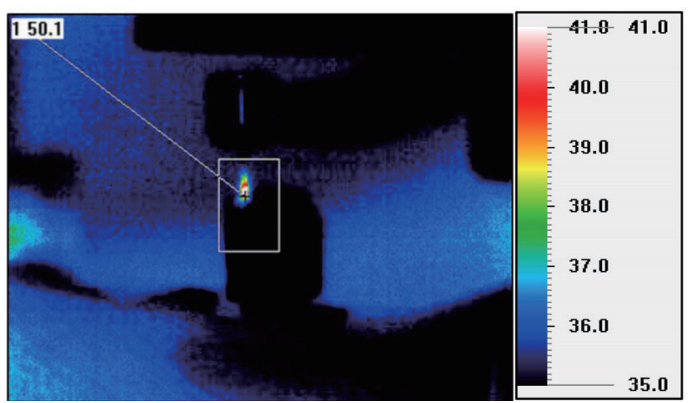

Fig. 21. (Color online) Cutting with cage-damaged rotor.

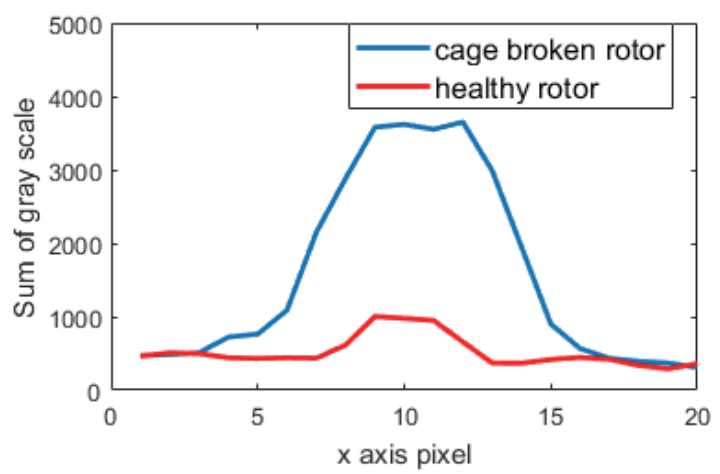

Fig. 22. (Color online) Histograms of the cutting thermal images.

\section{Conclusions}

In this research, signal analysis techniques were used with the FFT, histograms, and CNN algorithms to detect the fault status of a dental handpiece under idling and drilling conditions. Various pressures of air and statuses of the rotor were examined. Experimental results indicated the condition of the dental handpiece when a typical dentist uses it with a handy mobile phone and a plug-in infrared device within $1 \mathrm{~min}$ is feasible in the near future. A measure for infrared fault diagnosis using the $\mathrm{CNN}$ was developed. Using the CNN information, in experiments, healthy, cage-broken, and collet-broken rotors were identified with a high classification rate within 30 and $60 \mathrm{~s}$, thus achieving diagnosis of the health status of the dental handpiece in a short time. The experimental results indicated that the infrared signals of thermal features were extracted with a good classification rate by using the $\mathrm{CNN}$, while a $100 \%$ classification rate was achieved when the learning NN nodes were well established. The deployment of the proposed method in a clinic is feasible. Heuristically, by training with more infrared images, the CNN can facilitate high-precision classification. The number of CNN layers affects the accuracy. The larger the training set, the faster the convergence and the higher the accuracy. In this work, we performed an experiment with idling for $30 \mathrm{~s}$. By comparing different pressures, we found that the accuracy of training results at $25 \mathrm{psi}$ is nearly $100 \%$. A systematic methodology for dental handpiece prognosis, health assessment, and determination of remaining useful life using long- and short-term memories will be developed in the future. 


\section{Acknowledgments}

The authors thank the Ministry of Science and Technology for financially supporting this research in part under Grant MOST 108-2221-E-018-013.

\section{References}

1 L. Zach and G. Cohen: Oral Surg. Oral Med. Oral Pathol. 19 (1965) 515. https://doi.org/10.1016/00304220(65)90015-0

2 A. Widodo, D. Satrijo, T. Prahasto, G. M. Lim, and B. K. Choi: Int. J. Rotating Mach. 2012 (2012) 1. https:// doi.org/10.1155/2012/847203

3 H. Liu, C. Bao, T. Xie, S. Gao, X. Song, and W. Wang: Infrared Phys. Technol. 96 (2019) 390. https://doi. org/10.1016/j.infrared.2018.08.028

4 O. Janssens, M. Loccufier, R. V. de Walle, and S. V. Hoecke: IEEE/ASME Trans. Mechatron. 23 (2018) 151. https: //doi.org/10.1109/TMECH.2017.2722479

5 Y. LeCun, L. Bottou, Y. Bengio, and P. Haffner: Proc. IEEE 86 (1998) 2278. http://doi.org/10.1109/5.726791

6 A. Krizhevsky, I. Sutskever, and G. E. Hinton: Commun. ACM 60 (2017) 84. https://doi.org/10.1145/3065386

\section{About the Authors}

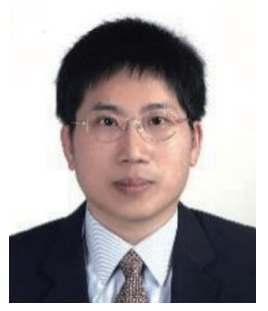

Yi-Cheng Huang received his B.S. and M.S. degrees in mechanical engineering from National Central University, Chung Li, Taiwan, in 1988 and 1990, respectively. He received his Ph.D. degree in mechanical engineering from Columbia University, New York, USA, in 1996. From 1996 to 1997, he worked as a senior engineer at the Equipment, Technology, and Mechanization Department of Philip Electronics Industry in Taiwan. Since 1997, he has been with the National Changhua University of Education, Changhua, Taiwan, where he is currently a distinguished professor in the Department of Mechatronics Engineering. In 2002, he worked as a visiting scholar at the University of Minnesota. His current research interests include iterative learning control, CNC machine tool control, prognosis of ball screw feed drive systems, signal processing, and machine learning.

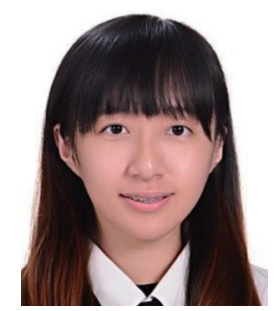

Pin-Jun Wang received her M.S. degree from the Department of Mechatronics Engineering, National Changhua University of Education, Changhua, Taiwan, in 2019. Her current research interests include the prognosis of dental handpiece systems, signal processing, and machine learning. 